\title{
ELISABETH RUCHAUD
}

\section{Le pèlerin chrétien vers Jérusalem Une construction de l'image de l'»autre«}

Le phénomène de mobilité ponctuelle qui participe à une définition de la dynamique pérégrinatoire est aussi un vecteur porteur d'informations qui a contribué à la construction d'une imagerie largement stéréotypée de l'autre musulman dans la pensée et l'imaginaire occidentaux.

Le pèlerinage chrétien est une expression des pratiques religieuses où le fidèle va à la rencontre du divin dans un lieu reconnu comme tel ${ }^{1}$. En tant que fait social complexe, il implique différentes composantes, d'un côté communes à toute démarche de pèlerinage et de l'autre spécifiques à chaque objectif. La peregrinatio est une démarche personnelle du jeune homme riche qui répond à l'appel du Christ de tout laisser pour le suivre ${ }^{2}$. À son image, le pèlerin abandonne, pour des raisons spirituelles, son propre milieu, toutes ses relations sociales et familiales, pour vivre un chemin tant physique que spirituel, comme un étranger, dans le but d'atteindre un lieu marqué par la manifestation du divin. Le peregrinus prend la route vers un ailleurs qui représente le lieu du mystère et du désir. Le terme même recouvre la double interprétation de la démarche de partir (per agere) et de son statut d'étranger circulant en marge du monde. Les routes suivies par les pèlerins étaient aussi le cadre de rencontres humaines, et les étapes dans les auberges, hospices ou monastères, l'occasion d'échanger des informations qui, par la suite, continuaient de circuler.

$\mathrm{Au}$ terme de sa pérégrination, le »marcheur de Dieu« entreprend une dernière démarche spirituelle: le récit, écrit ou oral, de l'ensemble de son voyage, de ses visites des Lieux saints, de ses expériences et de ses épreuves. En tant que phénomène exceptionnel, le compte-rendu a posteriori de la pérégrination est une suite et conclusion logique de la démarche ${ }^{3}$. Le fait de relater son expérience de voyage et sa découverte des Lieux saints, d'en faire profiter ceux qui ne peuvent prendre ou n'ont pas encore pris la route, appartient encore aux obligations incombant au pèlerin à son retour. L'éloignement géographique de la Terre sainte favorisa le développement de l'intérêt des voyageurs sur d'autres éléments que la seule sacralité des lieux. De par son statut de voyageur qui découvre de nouvelles contrées, le pèlerin est avant tout attentif à

1 Aryeh GRABOÏs, Le pèlerin occidental en Terre sainte au Moyen Âge, Paris, Bruxelles 1998, p. $12-17$.

2 Mt 16,24.

3 Les diverses démarches à entreprendre afin de pouvoir partir, sans oublier les cérémonies accompagnant le départ en pèlerinage, témoignent du caractère exceptionnel du phénomène qui situe le pèlerin hors de la société à laquelle il appartient. Cf. Elisabeth RuCHAUD, Peregrinatio et voyage au début du Moyen Âge. Jérusalem et la Terre sainte, dans: Mobilität: Von den Ursprüngen der Reiselust bis zu ihrem Ende in der vermessenen Welt. Deutsch-französisches Graduierten-Kolloquium 2009, Stuttgart, à paraître. 
l'environnement physique des régions qu'il traverse, environnement qui reste pour lui totalement indissociable de la sacralité du pays et donc de sa démarche. Dans un premier temps, les récits de pèlerins s'attardent volontiers sur des descriptions souvent enthousiastes du lac de Tibériade, du Jourdain ou des principaux monuments de Jérusalem ${ }^{4}$, sans oublier la mention de la présence de communautés chrétiennes ${ }^{5}$. Il faut néanmoins attendre le XIII ${ }^{\mathrm{e}}$ siècle pour voir se dessiner chez certains un intérêt plus ou moins marqué pour l'environnement humain qui appartient, quant à lui, au monde du profane. Ces textes ont été rédigés pour la plupart par les pèlerins eux-mêmes au retour de leur voyage, par un clerc de leur entourage pour certains, ou par des auteurs compilant des récits oraux parfois longtemps après le pèlerinage en lui-même. On trouve aussi quelques mentions sporadiques de départs et de retours plus ou moins développées dans des chroniques locales. Il nous faut cependant souligner que la majorité des récits, anonymes ou non, encore à notre disposition se contente d'une énumération des sites et églises visités au cours du périple. Seuls quelques-uns l'agrémentent d'une description personnelle ou, souvent, empruntée à d'autres relations faisant autorité en la matière. Ce n'est que plus tard que de véritables traités sur la Terre sainte, insistant sur l'environnement tant physique qu'humain, commencent à circuler'6.

Le pèlerin, de par le mouvement migratoire qu'il entreprend, se trouve au cœur d'un processus de transfert d'informations direct ou indirect. L'analyse de son implication dans le processus montre qu'il s'agit d'un mécanisme tant conscient qu'inconscient. L'objectif premier de tout pèlerin reste avant tout la poursuite de sa quête spirituelle jusqu'au lieu où peut et doit s'exprimer sa dévotion. Dans le cas de Jérusalem, il s'agit bien évidemment du Saint-Sépulcre, métonymie de la ville où se concrétise le centre spirituel de la terre et se joignent expérience personnelle et héritage biblique. Par extension, lui sont associés les hauts lieux de la prédication prophétique et évangélique.

Au cours du Moyen Âge, une évolution se fait sentir dans le genre des récits de pèlerinage. Le récit est tout d'abord centré sur la motivation religieuse initiale et dont la piété s'exprime à l'arrivée par les diverses dévotions dans les Lieux saints. Les remarques et les descriptions qui l'enrichissent demeurent toujours en lien avec la quête spirituelle. Par la suite, les annotations et observations émaillant le récit, plus détachées

4 Sans être exhaustif, on peut citer quelques récits exemplaires reprenant des descriptions géographiques des contrées traversées sur le chemin vers ou depuis Jérusalem. En 383, Égérie, journal de voyage, éd. Pierre MARAval, Manuel Cecilio DíAZ y DíAZ, Paris ${ }^{2} 2002$; vers 700, Bède le Vénérable, Liber de locis sanctis, éd. Paul GEYER, Turnhout 1965 (Corpus Christianorum Series Latina, 175), p. 251-280, traduction dans: John WILKINSON, Jerusalem Pilgrims before the Crusades, Warminster 2002, p. 216-230; vers 870, Bernard le Moine, Descriptiones, dans: Titus TOBLER (dir.), Descriptiones Terrae Sanctae, Munich 1874, traduction dans: WiLKINSON, Jerusalem Pilgrims, p. 261-269.

5 Willibald (VII ${ }^{\mathrm{e}}$ siècle) fait ainsi état de rencontres avec d'autres communautés chrétiennes en Palestine et en particulier avec des moines. Voir Andreas BAUCH, Pilgerreise Willibalds ins Heilige Land, dans: Wolfdietrich FisCHER, Jürgen SCHNEIDER (dir.), Das Heilige Land im Mittelalter. Begegnungsraum zwischen Orient und Okzident, Neustadt an der Aisch 1982, p. 1318, plus spécifiquement p. 15-16.

6 GRABOÏs, Le pèlerin occidental (voir n. 1), p. 38-44. 
du caractère exclusivement sacré de la Terre sainte, tendent à s'intéresser aux espaces géographiques et aux sociétés humaines rencontrées.

En marge des motivations uniquement religieuses, ces récits de pèlerins sont aussi des sources d'information non négligeables. À travers leur étude, plusieurs types d'informations pourraient être et ont déjà été analysés: données économiques, géographiques $^{7}$, moyens mis à disposition pour le voyage, routes empruntées ${ }^{8}$, relations diplomatiques, événements politiques ${ }^{9}$, risques encourus, etc. Notre propos est ici de nous intéresser plus spécifiquement à la représentation de l'autre, en tant que nonchrétien et non-occidental, et en particulier à la construction d'une image de l'autre musulman. L'élément juif a quant à lui été largement ignoré et minoré par les pèlerins, à l'exception de quelques mentions sporadiques de présence de communautés pour des fêtes ou des grands rassemblements, ou d'individus isolés. Ils n'apparaissent qu'à travers des considérations théologiques qui mettent sur un pied d'égalité contemporains et ancêtres de l'époque du Christ. Cette absence quasi-totale des juifs en Terre sainte s'explique par le fait que, considérés comme déicides, ils ont été déchus de leur droit à la Terre promise et sont donc supposés ne plus se trouver en Terre sainte ${ }^{10}$.

À travers l'analyse, nécessairement rapide, de deux récits de pèlerins, l'un du $\mathrm{VIII}^{\mathrm{e}}$ siècle et l'autre du début du XIII ${ }^{\mathrm{e}}$, nous tenterons de percevoir l'équilibre se dessinant entre une image archétypale et le plus souvent caricaturale de l'autre musulman et le ressenti plus direct des pèlerins. Le choix de ces deux récits, éloignés dans le temps et différents dans le fond, permet d'établir une certaine forme de renouvellement s'opérant chez le pèlerin dans son appréhension du monde extérieur non-chrétien ${ }^{11}$.

7 Ibid., p. $117-136$.

8 Voir Françoise MiCHEAU, Les itinéraires maritimes et continentaux des pèlerinages vers Jérusalem, dans: Occident et Orient au $\mathrm{X}^{\mathrm{e}}$ siècle. Actes du $\mathrm{IX}^{\mathrm{e}}$ congrès de la société des historiens médiévistes de l'enseignement supérieur public (Dijon, 2-4 juin 1978), Paris 1979, p. 79-112.

9 Les diverses péripéties émaillant le trajet du grand pèlerinage allemand de 1064-1065 sont un exemple des difficultés de certains pouvoirs locaux à maintenir une route sûre, et ce malgré la conversion des Hongrois (fin du $\mathrm{X}^{\mathrm{e}}$ siècle). De même, les multiples démêlés avec les pouvoirs musulmans locaux témoignent des obstacles rencontrés par les pèlerins. Voir Annales Altahenses maiores, 8, a. 1065, éd. Wilhelm von GIESEBRECHT, Edmund von OEFELE, Hanovre 1868 (Monumenta Germaniae Historica, Script. rer. Germ., [4]), p. 815-817.

10 Suzanne CONKLIN AKBARI, Idols in the East. Representations of Islam and the Orient, 1100 1450, Ithaca 2009, plus spécifiquement le chapitre 3: The Place of the Jews, p. 112-154, où l'auteur revient sur la place de l'élément juif en Orient entre chrétiens et musulmans. D'un point de vue géographique, elle souligne que les communautés juives d'Israël ont été encloses dans des espaces définis en dehors de Jérusalem (p. 115).

11 Dans un ouvrage récent, Armelle Leclercq propose une analyse comparée des sources latines et arabes sur la première croisade pour un regard croisé sur la perception de l'autre: Armelle LECLERCQ, Portraits croisés. L'image des Francs et des musulmans dans les textes sur la première croisade, Paris 2010. On regrettera cependant l'absence du récit de Thietmar dans son analyse, récit qui aurait pourtant été un exemple pertinent pour sa démonstration. L'ouvrage de Norman Daniel reste aussi une référence en ce qui concerne la construction d'une image de l'islam dans l'Occident médiéval, voir: Norman DANIEL, Islam and the West. The Making of an Image, Oxford 2000. 


\section{WILLIBALD, UN RÉCIT DU VIII ${ }^{\mathrm{e}}$ SIÈCLE}

Comme nous l'avons déjà relevé, les récits connus les plus anciens ne mentionnent que très sporadiquement et de façon tout à fait marginale les sociétés rencontrées. Même si certains éléments relatifs aux églises orientales et au monachisme sont plus ou moins disséminés dans le récit, comme par exemple chez Bernard le Moine ${ }^{12}$, ces »descriptiones « avaient pour fonction principale de rendre compte de la démarche, des épreuves qui attendaient le voyageur courageux, du cheminement tant physique que spirituel et, une fois arrivé, de décrire les Lieux saints visités. En revanche, en aucun cas il n'avait pour objectif la description des sociétés et des habitants croisés qui appartenaient quant à eux à la sphère du profane. Une distinction précise existe en effet dans les récits entre ce qui relève du sacré - géographie du sacré, monuments, communautés chrétiennes locales, épreuves... - et ce qui appartient au monde profane - sociétés, modes de fonctionnement... - et ne peut, à ce titre, être un objet d'étude ou même seulement de description. Le récit de Willibald illustre cette approche que l'on pourrait qualifier de spurement spirituelle` de la relation de pèlerinage ${ }^{13}$.

Willibald, évêque d'Eichstätt, n'a pas écrit lui-même son récit mais l'a raconté de vive voix à des rédacteurs. Le texte fut vraisemblablement rédigé du vivant du saint, entre 760 et 780, et est connu sous deux versions. La première est l'œuvre d'une religieuse du monastère d'Heidenheim (près d'Ulm) du nom de Huneberc (ou Hugeburc). La seconde serait celle d'un diacre que Huneberc nous dit avoir assisté dans sa tâche. Malheureusement, la religieuse omet de nous préciser la date exacte de cette rédaction $^{14}$. C'est tout juste âgé d'une vingtaine d'années, vers 720 , que le jeune Willibald accompagne son frère et son père en pèlerinage à Rome. Ce dernier décédant en Italie, Willibald est alors pris d'un désir de pousser plus loin encore son périple et de se rendre jusqu'à Jérusalem. Toujours flanqué de son frère et de ses six autres compagnons dont on ignore l'identité, il va rejoindre, non sans difficulté, Jérusalem via Naples, la Sicile, l’Égypte et Chypre.

12 Son récit nous renseigne aussi sur les conditions de circulation en mer au $\mathrm{X}^{\mathrm{e}}$ siècle, les contrôles imposés, le système de taxation qui pèse sur son voyage, etc. Voir: Danielle RÉGNIERBOHLER (dir.), Croisades et pèlerinages. Récits, chroniques et voyages en Terre sainte, $\mathrm{XII}^{\mathrm{e}}-$ $\mathrm{XVI}^{\mathrm{e}}$ siècle, Paris 1997, p. 916-927.

13 Huneberc, Peregrinatio sive Hodoeporicon ad Terram Sanctam S. Willibaldi, éd. Titus ToBLER, Auguste MOLINIER, Itinera Hierosolymitana et Descriptiones Terrae Sanctae bellis sacris anteriora, Osnabrück ${ }^{3} 1966$, p. 239-281; WILKINSON, Jerusalem Pilgrims (voir n. 4), p. 233-251. Pour une analyse plus complète voir: Klaus GUTH, Die Pilgerfahrt Willibalds ins Heilige Land (723-727/729). Analyse eines frühmittelalterlichen Reiseberichts, dans: Sammelblatt des Historischen Vereins Eichstätt 75 (1982), p. 13-28. Sa relation, la seule connue pour le VIII ${ }^{\mathrm{e}}$ siècle, permet de faire le lien entre les récits d'Arculf (670) et de Bernard le Sage (870).

14 D'après certains éléments du récit, il semble que Willibald était encore vivant lorsqu'elle entreprend son ouvrage. Ce dernier étant mort en 787, les indications fournies permettent d'avancer une fourchette large de datation entre 760, date de l'installation de sa sœur Walburge à Heidenheim, et 780, après la mort de celle-ci qu'il fait enterrer à Heidenheim. RÉGNIERBOHLER, Croisades et pèlerinages (voir n. 12), p. 893-896. 
Son témoignage constitue, par sa construction, un exemple de récit oscillant entre découverte de la Terre sainte et intervention du Merveilleux et des miracles. Si le pèlerin s'attache à décrire les Lieux saints, les monuments ${ }^{15}$ et même les communautés chrétiennes qu'il a pu croiser sur son itinéraire, les sociétés autochtones et leurs coutumes ne sont que marginalement mentionnées ${ }^{16}$. En effet, Willibald revient avec force détails sur son bain dans le Jourdain ${ }^{17}$, rappel du baptême du Christ par Jean-Baptiste, il décrit les agencements des églises de Jérusalem et plus particulièrement son passage au Saint-Sépulcre. Il relate même sa rencontre avec un lion »horrible, rugissant et ouvrant la gueule comme pour les saisir et les dévorer « ${ }^{18}$ sur leur route de retour vers le Liban. Mais, comme il le souligne par la suite, le lion »par le secours du Dieu très bon et tout puissant, partit dans une autre direction, leur laissant la voie libre « ${ }^{19}$.

En revanche, il ne s'étend guère sur sa période de captivité à Émèse (Syrie). De passage dans la ville pour visiter la basilique dédiée à saint Jean-Baptiste, Willibald et ses compagnons sont arrêtés par les autorités musulmanes pour ne pas avoir pu présenter leur sauf-conduit en règle. Si les conditions assez douces de leur incarcération sont mentionnées ${ }^{20}$, le narrateur ne s'attarde pas à décrire l'environnement humain côtoyé pendant cette période ni le mode de fonctionnement de la cité. Les locaux sont génériquement désignés par le terme de sarracenus (sarrasin) et la libération des pèlerins intervient sur l'ordre »du roi des sarrasins, appelé Mirmumni« ${ }^{21}$, ce qui renvoie sans doute au titre des califes amīr al-mu'mininn (chef des croyants). Le récit revient en revanche plus longuement sur la bonté de leurs bienfaiteurs, un marchand prêt à payer leur rançon et un dignitaire espagnol de la cour califale, sans pour autant développer leur rôle dans la société ou leur part active à leur libération. De même, on ne trouve

15 Dans une étude récente, Rodney Aist a entrepris d'analyser les descriptions de Willibald concernant Jérusalem et d'en tirer les conclusions concernant la topographie religieuse de la ville au VIII ${ }^{\mathrm{e}}$ siècle. Voir Rodney AIST, The Christian Topography of Early Islamic Jerusalem: The Evidence of Willibald of Eichstätt (700-787 CE), Turnhout 2009.

16 Andreas Bauch dans son analyse du texte souligne les quelques mentions qui sont faites des »sarrasins«, soulignant qu'elles sont toujours faites en lien avec un élément chrétien avéré (une église, une communauté, etc.). Voir BAUCH, Pilgerreise Willibalds ins Heilige Land (voir n. 5), p. 16-17.

17 Huneberc, Peregrinatio sive Hodoeporicon (voir n. 13), chap. 15, p. 261-262; WILKINSON, Jerusalem Pilgrims (voir n. 4), chap. 15, p. 240.

$18 »[\ldots]$ unus leo, qui, aperto ore, rugiens rancusque«, dans: Huneberc, Peregrinatio sive Hodoeporicon (voir n. 13), chap. 38, p. 270; WILKINSON, Jerusalem Pilgrims (voir n. 4), chap. 28, p. 247.

19 »Ast ille cito, omnipotentis altithroni Dei adminiculo, in alliam partem divertit, et latabat illis viam, ut pergerent«, dans: Huneberc, Peregrinatio sive Hodoeporicon (voir n. 13), chap. 28, p. 270; WILKINSON, Jerusalem Pilgrims (voir n. 4), chap. 28, p. 247.

20 Huneberc, Peregrinatio sive Hodoeporicon, (voir n. 13), p. 257-260; WiLKINSON, Jerusalem Pilgrims (voir n. 4), chap. 12, p. 236-239. La captivité semble être datée de 724.

$21 »[\ldots]$ omnes simul venerunt coram rege Sarracenorum, cui nomen Mirmumni «, dans: Huneberc, Peregrinatio sive Hodoeporicon (voir n. 13), chap. 12, p. 259; WiLKInSON, Jerusalem Pilgrims (voir n. 4), chap. 12, p. 237. 
qu'une mention particulièrement succincte du marché de la ville 22 que le saint et ses compagnons de captivité traversent cependant quotidiennement pour se rendre à l'église. Dans le cours du récit, cet épisode n'intervient, au même titre que celui du lion, que pour montrer la foi rayonnante et la dignité des pèlerins qui s'accommodent de leur captivité et du retard pris sur leur chemin. Leur piété visible, ainsi que leur apparence physique ${ }^{23}$, leur attire invariablement les regards, la sympathie et l'admiration.

Les récits oraux des pèlerins du $\mathrm{IX}^{\mathrm{e}}$ siècle et des siècles suivants, récits dont on peut trouver une trace à travers les chroniques, insistent quant à eux sur les persécutions infligées par les sarrasins qualifiés de païens, les attaques de brigands, les emprisonnements arbitraires coupant l'élan du pèlerin, les taxes jugées abusives réclamées comme droit de passage, ou enfin les diverses profanations dont la plus emblématique reste celle du mont du Temple où aurait été installée une statue de Mahomet ${ }^{24}$. Il devait en réalité s'agir des vestiges sculptés de l'ancien temple de Jupiter que les observateurs occidentaux, dans leur méconnaissance de la religion islamique, considéraient comme étant une statue de Mahomet et une image monumentale de leur idolâtrie et de leur polythéisme ${ }^{25}$. Cette information est reprise chez presque tous les chroniqueurs ${ }^{26}$ témoins de la première croisade, à l'exception d'Albert d'Aix qui n'en fait pas mention. Seul Guibert de Nogent, qui n'a quant à lui jamais été à Jérusalem, en sa qualité d'>historien`réfute l'assertion et affirme qu'il s'agit d'une calomnie ${ }^{27}$. Ce témoignage nous

${ }^{22}$ La mention du marché semble ici n'être qu'un élément topographique donné pour situer l'église dans la ville. On sait seulement que les captifs pouvaient y »acheter ce qui leur faisait plaisir«, ce qui témoigne sans doute d'un lieu de commerce relativement grand et achalandé.

23 Ils sont décrits comme »qui iuvenes et decori et vestium ornatu induti erant bene«, Huneberc, Peregrinatio sive Hodoeporicon (voir n. 12), chap. 12, p. 259; Wilkinson, Jerusalem Pilgrims (voir n. 4), p. 237.

24 Cette confusion entre les sculptures antiques qualifiées d'idolâtres et l'hypothétique représentation de Mahomet n'apparaît véritablement que dans les sources des croisades et n'est pas mentionnée par les voyageurs antérieurs. Les textes les plus révélateurs ont été compulsés et étudiés dans: Xenia MuRatova, Western Chronicles of the First Crusade as Source for the History of Art in the Holy Land, dans: Jaroslav FoLDA (dir.), Crusader Art in the Twelfth Century, Oxford 1982, p. 47-62.

25 À propos de la vision idolâtre de l'islam par l'Occident médiéval et les histoires rapportées à ce sujet voir: Jean FLORI, Croisade et chevalerie, $\mathrm{XI}^{\mathrm{e}}-\mathrm{XII}{ }^{\mathrm{e}}$ siècles, Paris, Bruxelles 1998, p. 163178; DANIEL, Islam and the West (voir n. 11), p. 338-343.

26 On peut citer notamment: Raoul de Caen, Gesta Tancredi, 129, dans: Recueil des historiens des croisades. Historiens occidentaux, vol. 3, Paris 1866, p. 695; Foucher de Chartres, Historia Iherosolymitana, I, 26, dans: Recueil des historiens des croisades. Historiens occidentaux, vol. 3, Paris 1866, p. 357; Baudri de Bourgueil, Historia Jerosolymitana, I, 4, dans: Recueil des historiens des croisades. Historiens occidentaux, vol. 4, Paris 1879, p. 13.

27 Guibert de Nogent, Gesta dei per Francos, I. 3. Pour la traduction française: Guibert de Nogent, Geste de Dieu par les Francs. Histoire de la première croisade, trad. Monique-Cécile GARAND, Turnhout 1998, p. 60. Dans ce chapitre, Guibert mentionne »Mathome« (Mahomet) comme d'après des on-dit et des éléments qui lui ont été rapportés. Mais il reconnaît aussi sa méconnaissance complète sur l'histoire de Mahomet et de sa nouvelle religion qui a »complètement détourné les Orientaux de la croyance au Fils et au Saint-Esprit«. Il reste cependant sceptique face à de nombreuses rumeurs dont on lui a fait part, ces dernières ne reposant pour lui sur rien de concret (notamment en ce qui concerne cette statue de Mahomet sur le mont du Temple). 
montre que Guibert de Nogent est prêt à relever et mettre en doute les assertions de son temps, mais aussi qu'il reste tout de même enclin à les employer dans le cadre d'une vérité idéologique qui l'emporterait sur l'exactitude objective de l'historien.

C'est le plus souvent à partir de ces données parcellaires et parfois volontairement détournées que tend à se dessiner, aux alentours de la première croisade, l'image de l'occupant de la Terre sainte comme étant celle d'un Oriens horribilis à combattre. Cette image semble encore trouver une confirmation, pour les chroniqueurs, dans les agissements du calife fatimide al-Hākim, qui ordonna, en 1009, la destruction totale du Saint-Sépulcre et interdit l'accès des Lieux saints aux pèlerins ${ }^{28}$.

À l'époque des croisades, l'islam se voit ainsi caractérisé par deux éléments négatifs: tout d'abord par l'idolâtrie, en particulier la figure de Mahomet vu comme un faux prophète et une fausse divinité; puis par la luxure, qui semble qualifier le mode de vie des Orientaux ${ }^{29}$. Ces traits répétés et déformés à de multiples reprises en sont venus à former la base d'un stéréotype fort, marquant durablement la vision occidentale du monde musulman, stéréotype dont les récits postérieurs, malgré une véritable >curiosité`, auront des difficultés à se détacher ${ }^{30}$.

\section{MAITTRE THIETMAR, UN CHANGEMENT DE MENTALITÉ}

L'intérêt marqué pour la religion et la société musulmanes du Proche-Orient n'est véritablement à placer qu'à partir du XIII ${ }^{\mathrm{e}}$ siècle $^{31}$. Il résulte du changement des mentalités occidentales légitimant le développement d'une certaine forme de curiosité auparavant considérée comme un vice - comme facteur de connaissance de l'»autre«, du reste du monde et par extension de soi-même. Cette évolution de la perception est sans doute à mettre en relation avec un renouveau de la philosophie et de la pensée

${ }^{28}$ Le fait est mentionné principalement chez Raoul Glaber (Historiae, Livre III, chap. VII,24, trad. Mathieu ARNOUX, Turnhout 1996, p. 182-183) et repris ensuite par Adémar de Chabanne. Dans une étude, John France a tenté de déterminer l'impact plus ou moins réel de cet événement en Occident. Il en est arrivé à la conclusion que mis à part Glaber, personne ou presque n'a répertorié le fait dans les chroniques locales. John FRANCE, Les origines de la première croisade: un nouvel examen, dans: Michel BALARD (dir.), Autour de la première croisade, Paris 1996, p. 45-56. Il est cependant indéniable que le fait a été repris dans l'argumentaire de la croisade, sans doute relayé par le monde byzantin qui avait entrepris une première reconstruction du Saint-Sépulcre sous Constantin Monomaque et avait demandé l'aide de l'Occident dans la région.

${ }^{29}$ L'ensemble de ces polémiques est repris dans: LECLERCQ, Portraits croisés (voir n. 11), p. 189228.

30 Daniel revient notamment longuement dans les premiers chapitres sur la construction de l'image de l'islam dans l'Occident médiéval et notamment sur cette construction comme mécanisme défensif, voir: DANIEL, Islam and the West (voir n. 11), p. 67-99.

31 Pour une étude plus poussée, voir John Tolan, Sons of Ishmael. Muslims Through European Eyes in the Middle Ages, Gainesville 2008. Dans cette étude, Tolan insiste plus particulièrement sur les sources du XIII ${ }^{\mathrm{e}}$ siècle et postérieures (notamment tout un chapitre sur Pierre le Vénérable, p. 46-65). 
grecques platoniciennes et aristotéliciennes, via le monde musulman ${ }^{32}$. À cela s'ajoute l'impact des nouveaux ordres prêcheurs comme celui des frères mineurs de François d'Assise, qui participe à une redécouverte des autres dans leur vocation prosélyte 33 . Malgré tout, les pèlerins de cette période, tous désireux de découvrir et de diffuser leur nouveau savoir, sont confrontés à une difficulté majeure. Il leur faut en effet pouvoir faire une distinction entre la Jérusalem idéalisée des récits évangéliques et les réalités matérielles de l'occupation musulmane. C'est ainsi qu'au début on trouve plus souvent des observations et des remarques concernant les pays limitrophes traversés qu'une analyse de l'état de la Terre sainte et de Jérusalem.

Le récit de Thietmar ${ }^{34}$ n'échappe pas à cette dichotomie. Il offre ici une première ébauche de découverte de l'autre en dehors de toutes considérations purement théologiques ou eschatologiques. Écolâtre allemand en pèlerinage vers Jérusalem en 1217, ce frère mineur a été l'un des premiers à mettre par écrit ses observations et ses impressions au cours de son périple, et notamment sur les occupants musulmans envers lesquels il éprouve une véritable curiosité. On ne sait malheureusement que peu de choses le concernant ${ }^{35}$, mais il est probable qu'il s'agisse de l'un des premiers frères mineurs à s'être rendu en Terre sainte, peu après la visite de saint François en Égypte ${ }^{36}$. Son compte rendu détaillé est aussi une importante source d'information concernant la situation de la Palestine en général et de Jérusalem en particulier, peu après la bataille de Haț̣īn (1187). Conscient des difficultés pour un pèlerin franc d'accéder aux Lieux saints, Thietmar n'hésite pas à se faire pousser la barbe et se vêtir en moine géorgien afin de circuler plus librement jusque dans des zones reculées ${ }^{37}$. Il livre alors une observation minutieuse non seulement de son environnement géographique, mais aussi de la société composite qu'il croise, à laquelle se mêle le classique récit des miracles dont il est le témoin ou qui lui sont rapportés. Ainsi Thietmar est-il fortement impressionné par les paysages qu'il croise sur sa route, et en particulier le désert et les mon-

32 Alain de LiBERA, La philosophie médiévale, Paris ${ }^{2} 2004$.

33 Dont John Tolan, Le saint chez le sultan: la rencontre de François d'Assise et de l'islam: huit siècles d'interprétation, Paris 2007; ID., Porter la bonne parole auprès de Babel: les problèmes linguistiques chez les missionnaires mendiants, $\mathrm{XIII}^{\mathrm{e}}-\mathrm{XIV}^{\mathrm{e}}$ siècle, dans: Peter von MOOS (dir.), Entre Babel et Pentecôte. Différences linguistiques et communication orale avant la modernité $\left(\mathrm{VIII}^{\mathrm{e}}-\mathrm{XVI}^{\mathrm{e}}\right.$ siècle), Berlin 2008, p. 533-547; Benjamin Kedar souligne quant à lui le rôle des ordres prêcheurs (franciscains et dominicains) qui reprennent le discours et l'intérêt pour l'islam après l'échec des croisades, voir Benjamin Z. KEDAR, Crusade and Mission: European Approaches towards the Muslims, Princeton 1984.

34 Magistri Thietmari peregrinatio, éd. J. C. M. LAURENT, Hambourg 1857, traduction française par Christiane DELUZ, Le pèlerinage de Maître Thietmar, dans: RÉGNIER-BOHLER, Croisades et pèlerinages (voir n. 12), p. 928-958.

35 Il est probablement originaire de Westphalie puisqu'il dit avoir rencontré des compatriotes de cette région prisonniers à Damas. Voir RÉGNIER-BOHLER, Croisades et pèlerinages (voir n. 12), p. 936-937.

36 TolAn, Le saint chez le sultan (voir n. 33).

37 RÉGNIER-BOHLER, Croisades et pèlerinages (voir n. 12), p. 940. Il s'agit en l'occurrence pour lui de se rendre, par la route de la côte, d'Acre au mont Carmel pour y vénérer le »corps de la bienheureuse Catherine d'où s'écoule une huile Sainte«. Il s'agit sans aucun doute du monastère de Sainte-Catherine sur le mont Sinaï. 
tagnes de Transjordanie ${ }^{38}$. Au cours de son séjour à Damas, il entend parler de captifs occidentaux, dont quelques compatriotes, mais, ne pouvant les rencontrer, il n'engage qu'un échange de lettres, et semble marqué par son impuissance à demander leur libération $^{39}$. Il semble enfin tout particulièrement fasciné par les communautés chrétiennes locales chez qui ils séjournent, retrouvant chez elles, lui semble-t-il, des éléments directement issus des premiers chrétiens ${ }^{40}$.

Esprit curieux de tout, il observe très attentivement les musulmans de Damas et de ses alentours, qui sont pour lui une véritable découverte. L'ensemble de sa description repose sur un mode dichotomique entre admiration et rejet. Il met ainsi en exergue un certain nombre de qualités dignes selon lui de louanges et même d'admiration de la part des chrétiens. La plus importante est la piété dont ils font preuve dans leur foi et sa pratique. Revenant sur la transformation en mosquée d'un ancien monastère dédié à saint Paul ${ }^{41}$, il décrit avec une certaine admiration les rites de purification par l'eau, qu'il compare à la confession, et l'appel à la prière quatre fois par jour par le »héraut«. L'application et la piété dont il est le témoin l'impressionnent favorablement et il va même jusqu'à regretter de ne pas les retrouver avec autant de force chez ses coreligionnaires. En revanche, après avoir décrit le statut de la femme, il condamne fermement la polygamie qu'il considère, à l'instar de l'ensemble des Occidentaux, comme un vice. De même, s'il admire le jeûne sévère du ramadan et sa stricte observance, il qualifie les plantureux repas suivant la rupture du jeûne d'orgies. Il en conclut, dans la lignée d'une imagerie bien implantée, que la religion islamique, malgré d'indéniables et remarquables qualités, comporte intrinsèquement un aspect vicieux et luxurieux qui en détourne le message premier ${ }^{42}$.

S'il est un des premiers récits connus à s'intéresser à la société musulmane et à en rapporter quelques éléments, ses descriptions demeurent avant tout superficielles, souvent erronées, toujours marquées par des stéréotypes antérieurs et tout à fait déformées. Elles connurent cependant un certain retentissement en Occident où tout ou partie de son récit fut repris à plusieurs reprises et assez largement diffusé. Il en vint même à devenir un modèle de relation de pèlerinage, en particulier pour ses descriptions et son analyse des communautés humaines et de la société musulmane qui furent réemployées à plusieurs reprises sans que leurs auteurs ne citent pour autant leur source 43 .

Malgré une véritable curiosité et un intérêt sans aucun doute sincère pour comprendre et connaître un monde >exotique « qui lui était totalement étranger, malgré une tentative d'objectivité dans son analyse des sociétés qu'il a pu rencontrer en Syrie et à Jérusalem hors de toute considération théologique ou exégétique, Thietmar reste pro-

38 Ibid., p. 934.

39 Ibid., p. 936-937.

40 Ibid., p. 937-939.

41 Ibid., p. 936.

42 Voir Aryeh GRABOÏs, La découverte du monde musulman par les pèlerins européens au XIII ${ }^{\mathrm{e}}$ siècle, dans: Al-Masaq 5 (1992), p. 29-46; GRABOÏs, Le pèlerin occidental (voir n. 1), p. $144-151$.

43 RÉGNIER-BOHLER, Croisades et pèlerinages (voir n. 12), p. 929-930. 
fondément imprégné par les stéréotypes antérieurs d'un Oriens horribilis idolâtre et luxurieux. Les renseignements qu'il a recueillis à propos du califat de Bagdad sont évasifs. D'un point de vue institutionnel, il rapproche le califat de l'Église catholique afin de faire comprendre l'autorité religieuse des califes, et va même jusqu'à qualifier ce dernier de »pape des Sarrasins«. Mais il change de discours concernant leurs comportements qui sont à ses yeux vicieux et dépravés, en particulier en ce qui concerne l'usage du harem ${ }^{44}$. Enfin, ses informations sur la vie de Mahomet tiennent en quelques lignes relativement vagues, reprenant là encore quelques idées communes répandues en Occident.

L'exposé le plus complet sur l'islam n'intervient finalement que près d'un siècle plus tard, sous la plume du dominicain florentin Ricoldo da Monte Croce (vers 1300) qui, parlant couramment l'arabe et lecteur averti du Coran, se trouvait à Bagdad où il put véritablement observer la société musulmane ${ }^{45}$. Son récit, qui mériterait une analyse particulière, demeure cependant un unicum au sein d'une littérature qui reste le plus souvent marquée par les stéréotypes et les préjugés et est même par la suite enrichie par l'imagerie fabuleuse de l'Orient.

\section{CONCLUSION}

En conclusion, il faut bien souligner qu'il ne s'agit ici que de remarques générales sur le genre de la relation de pèlerinage et les informations sur l'Orient que l'historien contemporain peut espérer y trouver et ce dans seulement deux récits.

Chaque pèlerin ayant sa propre expérience personnelle du voyage et de la rencontre avec le monde musulman, chaque relation, malgré des éléments communs, en offrira une vision plus ou moins particulière. Le récit de Willibald, s'il n'analyse pas la société musulmane, ne véhicule pas pour autant une image véritablement négative des geôliers qui paraissent être plutôt considérés comme une entité presque négligeable. L'élément musulman n'est développé dans le cours du récit qu'en tant que péripétie, au même titre que les tempêtes maritimes ou les attaques, une épreuve ne venant que compléter le déroulement du pèlerinage en marge de la découverte des Lieux saints. Il faut aussi prendre en considération que Willibald relate son expérience dans les années 720 , une période à laquelle le monde arabo-musulman est encore véritablement mal connu en Occident.

Chez Thietmar en revanche, une ébauche d'analyse du monde profane est sensible mais pas encore véritablement aboutie. S'il pose un regard qu'il veut objectif sur le monde qui l'entoure au cours de sa pérégrination vers Jérusalem, son récit traduit

44 Ibid., p. 940.

45 Voir Marie-Thérèse D'AlvernY, La connaissance de l'islam en Occident du XI ${ }^{\mathrm{e}}$ siècle au milieu du XIII ${ }^{\mathrm{e}}$ siècle, dans: L'occidente e l'Islam nell'alto medioevo, Settimane di Studi di Spoleto, t. 7, Spolète 1964, p. 577-602; René KAPPLER (dir.), Ricoldo da Monte Croce Pérégrination en Terre sainte et au Proche Orient: texte latin et traduction. Lettres sur la chute de Saint-Jean d'Acre: traduction, Paris 1997. 
encore un certain nombre de préjugés et de méconnaissances tant de la société que de la religion qu'il découvre.

Ainsi, même si une évolution certaine tend à se faire sentir dans le genre littéraire, l'étude approfondie de ces deux récits, étude tout juste esquissée ici, montre combien la construction de l'image de l'autre par les pèlerins chrétiens occidentaux, malgré des ajustements, des descriptions plus précises et des connaissances accrues au fil du temps, reste toujours marquée par des bases fondées sur des récits anciens et un phénomène de confrontation.

À travers ces deux exemples, mon but initial n'était pas de dire que la représentation négative des musulmans a seulement été une construction mentale à mettre au crédit des pèlerins occidentaux. Au contraire! Ces derniers n'ont finalement que repris et développé ce qui était déjà considéré à l'époque comme étant une vérité idéologique d'un islam luxurieux et idolâtre. Cette image s'avère être une construction progressive formée en différentes couches autour des relations complexes et conflictuelles de communautés rivales se côtoyant sur des marges disputées. Les pèlerins médiévaux d'avant la première croisade, en tant que `témoins directs`, n'ont fait que participer à la diffusion de cette première vision. Avec eux, ce que l'on pourrait définir comme l'>élément musulman devient une péripétie du récit, une étape presque obligatoire qui démontre toute la difficulté de la route et qui vise à fortifier et éprouver la foi du fidèle pendant son chemin vers Jérusalem. Il n'est toutefois pas possible de déterminer une seule et unique image de l'autre musulman à partir des seuls témoignages de pèlerins. Cette construction est le fruit d'une multiplicité d'images qui proviennent de différents horizons et qui se diffusent à plus ou moins grande échelle en Occident.

Par la suite et malgré une véritable curiosité à l'égard des éléments extérieurs dont témoignent les récits postérieurs, l'image de l'autre reste profondément ancrée dans ce premier stéréotype encore renforcé par les difficultés politiques. Une vision dichotomique entre Oriens horribilis et fascination de l'exotisme permet d'amorcer une dissociation entre les deux visions pas encore totalement intégrée. 